\title{
Online Learning Modules in Anatomical Sciences: Effective Sources for Continued Learning for Medical Undergraduates During the Unprecedent COVID-19 Pandemic
}

\author{
Srinivasan Viveka ${ }^{1^{*}}(\mathbb{D})$, Nagavalli Basavanna Pushpa ${ }^{2}$ (D) Kumar Satish Ravi ${ }^{3}$ (D)
}

\begin{abstract}
Introduction. During the COVID-19 pandemic-induced lockdown, the selection of simple and authentic online material among plethora of web content is difficult for both students and teachers. This forces students and teachers to explore various avenues of learning.

The objective of this research was to evaluate free open-access anatomy e-learning resources in accordance with required standard learning outcomes for medical students.

Methods. During February 2021, an extensive search for online modules for learning anatomy across six Massive Open Online Courses, including edX, Coursera, Udemy, Khan Academy, Canvas and FutureLearn, along with Google and YouTube was conducted. Courses or modules on e-learning platforms, YouTube channels, standalone videos, anatomy atlases, 3D models were considered as learning resources and evaluated. Online materials were classified as structured learning resources if they had a defined syllabus, time duration and instructional design. Resources lacking these characteristics were considered as unstructured ones.

Results. Twenty structured learning courses were identified on the Udemy (6 courses), Coursera (3 courses), edX ( 2 courses), FutureLearn and Khan academy platforms. Learning resources available through Swayam Prabha were aligned with the defined syllabus and video lectures. The content hosted within Clinical Anatomy, Medvizz and Kenhub was eye-catching. Thirty-two YouTube channels offering standalone learning material were identified. Seven resource materials, other than YouTube channels, offered anatomy learning material in the form of charts and tables. Four websites noted to have 3D interactive learning content regarding gross anatomy.

Conclusions. During the pandemic-induced lockdown, the list presented in the study may act as guide in selection of the simplest and best materials for those teaching and learning anatomy in medical undergraduate courses. However, in most cases, there is no alignment with standard learning outcomes as defined by medical education regulatory authorities.
\end{abstract}

Keywords

Anatomy Learning; Medical Education; E-Learning; Online Resources; Structured Courses; Youtube Channels

${ }^{1}$ Department of Anatomy, Azeezia Institute of Medical Sciences and Research, Kollam, Kerala, India

${ }^{2}$ Department of Anatomy, JSS Medical College, Mysore, Karnataka, India

${ }^{3}$ Department of Anatomy, All India Institute of Medical Sciences (AIIMS), Rishikesh, Uttarakhand, India

${ }^{*}$ Corresponding author: vivekabharathi@gmail.com

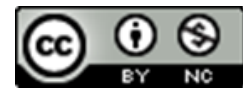

Copyright @Srinivasan Viveka, Nagavalli Basavanna Pushpa, Kumar Satish Ravi, 2021

\section{Introduction}

The COVID-19 pandemic has forced most of the medical institutes to conduct online classes [1,2]. With the onset of second wave and unprecedent circumstances, the need for comprehensive data of online learning material for a healthcare profes- sional is deeply felt. There are studies on evaluating e-learning among health professionals $[3,4]$. A meta-analysis (2008) of 117 such studies indicated that internet-based learning leads to a significant accumulation of knowledge [5]. Internetbased learning helps medicos improve their knowledge, as well as develop skills, behavioural and patient care competen- 
cies [5]. Instructional design focused on interactivity, practice exercises, repetition and feedback improves internet-based learning [6]. This has led to the recommendation that a wide range of internet-based education may be used effectively in health-related education [7].

Rapid social and educational communication is possible with modern social networking technologies [8]. The incorporation of such powerful online platforms for medical education has been evaluated in many previous studies $[9,10]$. There is also evidence that internet-based educational programs can be more effective than traditional teaching and learning programs as they are more convenient, have the ability to reach a wider audience, allowing each student to progress at their own speed [11-13]. The effectiveness of any internetbased learning depends on the quality of information provided $[10,14,15]$. There is a possibility that the information provided might be misleading, underrepresented, grossly generalized, or even false. Even though there are a lot of web sources to offer medical information, not all of them are valid and reliable. In addition, access to these resource by a medical student depends on convenience and requirements [16]. During the COVID-19 pandemic-induced lockdown, the selection of simple and authentic online material among plethora of web content is difficult for both students and teachers. Self-directed learning (SDL) is gaining importance in the competency-based curriculum. This forces students and teachers to explore various avenues of learning.

The objective of this research was to evaluate free openaccess anatomy e-learning resources in accordance with required standard learning outcomes for medical students.

\section{Materials and Methods}

\section{Search Strategy}

During February 2021, an extensive search for online modules for learning anatomy across six Massive Open Online Courses (MOOCs), including edX, Coursera, Udemy, Khan Academy, Canvas and FutureLearn, along with Google and YouTube was conducted. The search was undertaken using the following terms "online learning modules for anatomy", "anatomy health education", "anatomy learning videos", "anatomy Elearning", "learn anatomy", "human anatomy for medical students". Anatomy courses or modules on e-learning platforms, YouTube channels, standalone videos, anatomy atlases, 3D models were considered as learning resources and evaluated in this study.

\section{Inclusion Criteria}

- Well-defined audience: resources intended for first-year medical students.

- Declared authorship: clearly mention of the author and current (or recent/previous) affiliations.

- Full or partially open-access resources.

- Authenticity: authored by medical doctors.

- Only gross anatomy and neuroanatomy resources.

\section{Exclusion Criteria}

- Resources that do not indicate the intended audience or are intended for public, patient education, general health education. Such resources are addressed to beginners and may not be readily useful in summative assessments.

- Material with no author, university name or affiliations.

- Resources available only in intranet of the universities.

- Resources concentrating exclusively on entrance exams (postgraduate exams or licentiate exams including NEET-PG, PLAB, USMLE).

- Anatomy resources concentrating mainly on histology and embryology.

- Resources overlapping with pathology and other medical specialties/disciplines.

\section{Classification of Learning Resources}

The process comprised subdividing the material into structured courses and unstructured resources (Fig. 1).

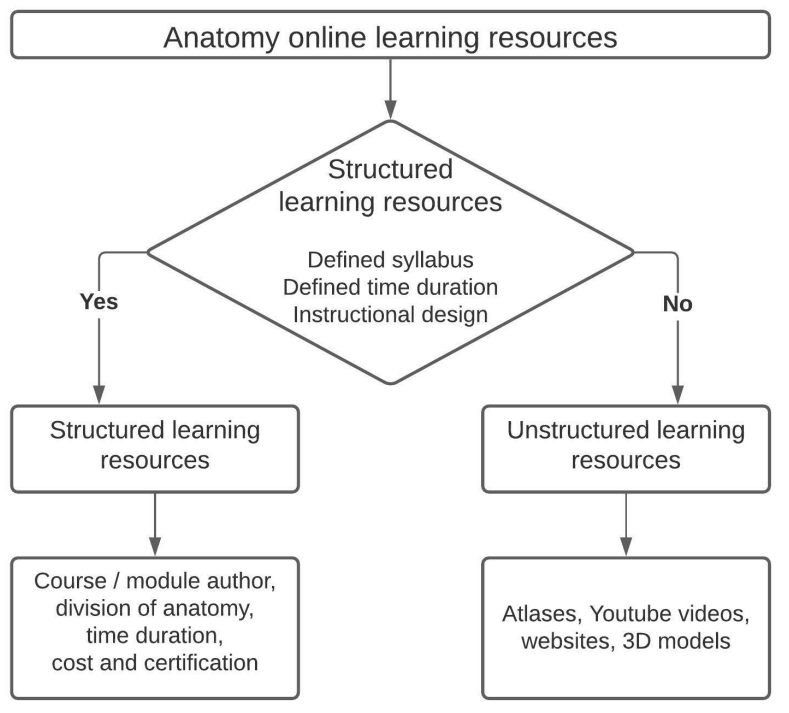

Figure 1. Schematic flowchart to classify learning resources as structured and unstructured.

Structured learning resources are learning resources with defined syllabus within the time frame. A built-in assessment of learning outcomes with learner feedback is an integral part of a well-structured instructional design. The following parameters were noted and tabulated: the name of the course; the website on which it is available; the name of the university/person/society offering the course/module; the syllabus (present - yes/no); anatomy section (gross anatomy, neuroanatomy, histology, embryology); duration in weeks; certification (offered - yes/no, if yes - the cost of certification).

Standalone resources without grouping / assessment / feedback / certification were classified as unstructured resources. 
Ungrouped standalone videos in any of the video sharing platforms constituted unstructured resources. The following parameters were noted and tabulated: the name of the website; the name of the university/person/society offering the resource; anatomy section (gross anatomy, neuroanatomy, histology, embryology.)

\section{Results}

\section{Structured Modules}

There were identified twenty-six structured courses in gross anatomy, including neuroanatomy, for medical students (Table 1). Six courses were excluded from the list - five courses had limited or partial access (the Anatomy Training Program co-sponsored by the American Association of Anatomists and the Anatomical Society; e-learning material for medical undergraduate training of Loyola University of Chicago; Online Anatomy Courses of West Virginia University School of Medicine; Human Anatomy Courses by McKinley and O'Loughlin) and Anatomy Guy E-Learning Course offered by the Radiological Society of North America (RSNA) was addressed to vertically integrated curriculum.

The National Programme on Technology Enhanced Learning (NPTEL), a project funded by the Ministry of Human Resource Development (MHRD), Government of India, has developed series of high-quality video lectures in most medical specialities, including anatomy [17]. The content is available under a Creative Commons Attribution-ShareAlike (CC BY-SA) licence through free Swayam Prabha DTH (Direct to Home) channels for education. Eminent anatomist, Dr. Vijaya Sagar, is the national co-ordinator for the compilation of these video lectures. As of February, 2021, 41 video lectures addressing to core competencies of anatomy learning are available [17]. Various eminent anatomists across India have recorded these video lectures.

The University of Michigan has designed a structured course for learning anatomy, called XSeries Program in Anatomy. It has been designed for 5 months, and certification is possible at the end of the course. The course runs a systems approach for learning anatomy [18]. The authors of the course are Kathleen Alsup, Glenn M. Fox, and Kelli A. Sullivan, all from University of Michigan. This course is available on both edX [18] and Coursera platforms [19]. The Hong Kong Polytechnic University has developed a Stroke-Based Learning Module for Anatomy. The authors claim that this is the first MOOC to teach human anatomy on a case-based scenario [20]. The edX platform hosts this course. On the same platform, Harvard University has designed a course with hypothetical injured patient to understand the anatomy of the musculoskeletal system [21].

Marco De Ruiter et al., the Leiden University Medical Centre, have developed a course Anatomy of the Abdomen and Pelvis; a Journey from Basis to Clinic. This course provides meaningful transition from basic concepts to applied anatomy [22]. Wesley Norman from Georgetown University has compiled region wise resources for learning anatomy on his personal website. In addition, he has designed tests at the end of each topic [23]. TeachMeSeries, supported by the University of Leicester, has developed TeachMeAnatomy Modules [24]. These modules are region wise and most of the content is available free or at a nominal price. Human Anatomy and Physiology Course on Khan Academy has system-based integrated modules in anatomy and physiology [25]. Charles Duncan, William B. Stewart and Shanta Kapadia from Yale University have created a course on Coursera platform called Anatomy of the Chest, Abdomen, and Pelvis [26]. Kenhub offers a series of learning modules, interactive images, flashcards, e-books and quizzes on its website [27]. Kenhub claims huge learning impact with their learning material. James Pickering, the University of Leeds, has designed a course Anatomy: Know Your Abdomen on FutureLearn platform [28].

Claudia Krebs, Monika Fejtek and Alexa Mordhorst, the University of British Columbia, have developed a comprehensive learning course Clinical Anatomy with attractive videos, interactive illustrations and radiology images, 3D constructions. The entire learning material is available free without even requirement of logging in (Creative Commons Attribution-NonCommercial-ShareAlike 4.0) [29].

Udemy platform offers three structured courses for learning anatomy. Robert Whitaker has designed a lecture course for learning clinically applied topographical anatomy Instant Anatomy: A Course in Human Anatomy [30]. The course content is more specific to Indian medical undergraduates. On the same platform, Ayman Taji has offered a course How to Remember Anatomy for a Lifetime [31]. Here the instructor has given all the mnemonics and techniques to help learner better remember the concepts.

Medvizz, a product of Proceum Pvt. Ltd., offers a range of courses, including anatomy. Dr. G. Bhanu Prakash Kulkarni with his comprehensive explanatory videos has covered most of gross anatomy topics. The entire self-study course, including videos, online tests and notes for quick reference, has been compiled and is available for medical undergraduates [32]. Attempts are being carried out to align the same with defined competencies under the new curriculum.

\section{Unstructured Learning Materials}

Thirty-two YouTube channels offering standalone learning material were identified. They are listed in Table 2 according to the increasing number of views (as of February 2021). Of special note were gross anatomy learning videos designed by eleven authors of Indian origin. The rest of the channels were owned by either foreign authors/institutions or anonymous authors.

Seven resource materials, other than YouTube channels, offered anatomy learning material in the form of charts and tables. Four websites noted to have 3D interactive learning content regarding gross anatomy (Table 3). Anatomylearning.com offers free 3D human models with more than 6, 000 anatomical details marked. These models are free to use for 
Table 1. Tabulation of structured online learning modules in anatomy.

\begin{tabular}{|c|c|c|c|c|c|c|c|}
\hline No & Course name & $\begin{array}{l}\text { Offered by (university or indi- } \\
\text { vidual) }\end{array}$ & $\begin{array}{l}\text { Anatomy } \\
\text { section }\end{array}$ & Time & Cost & $\begin{array}{l}\text { Certifi- } \\
\text { cation }\end{array}$ & URL \\
\hline 1 & $\begin{array}{l}\text { NPTEL-DTH } \\
\text { Swayamprabha Video } \\
\text { Lectures in Anatomy }\end{array}$ & $\begin{array}{l}\text { Anatomy professors from var- } \\
\text { ious institutions of India; } \\
\text { T. Vijaya Sagar, national } \\
\text { co-ordinator, National Pro- } \\
\text { gramme on Technology En- } \\
\text { hanced Learning }\end{array}$ & Gross anatomy & Self-paced & Free & No & http://dth.ac.in/medical/ \\
\hline 2 & $\begin{array}{l}\text { XSeries Program in } \\
\text { Anatomy }\end{array}$ & University of Michigan & Gross anatomy & 5 months & Free & USD176 & $\begin{array}{l}\text { https://www.edx.org/xseries/ } \\
\text { anatomy }\end{array}$ \\
\hline 3 & Human Anatomy & $\begin{array}{l}\text { Hong Kong Polytechnic Uni- } \\
\text { versity }\end{array}$ & $\begin{array}{l}\text { Stroke-based } \\
\text { case scenario }\end{array}$ & 8 weeks & Free & USD140 & $\begin{array}{l}\text { https://www.edx.org/course/ } \\
\text { human-anatomy }\end{array}$ \\
\hline 4 & Anatomy Lesson & Wesley Norman & $\begin{array}{l}\text { Region wise } \\
\text { anatomy }\end{array}$ & Self-paced & Free & No & http://www.wesnorman.com/ \\
\hline 5 & $\begin{array}{l}\text { Human Anatomy } \\
\text { Learning Modules }\end{array}$ & $\begin{array}{l}\text { Virginia Lyons and Nancy } \\
\text { McNulty, Geisel School of } \\
\text { Medicine at Dartmouth }\end{array}$ & Gross anatomy & Self-paced & Free & No & $\begin{array}{l}\text { https://www.dartmouth.edu/ } \\
\sim \text { anatomy/HAE/index.html }\end{array}$ \\
\hline 6 & Kenhub & Kenhub team & $\begin{array}{l}\text { Gross anatomy } \\
+ \text { histology }+ \\
\text { medical } \\
\text { imaging }\end{array}$ & Self-paced & $\begin{array}{l}\text { USD21/ } \\
3 \text { months }\end{array}$ & No & https://www.kenhub.com/ \\
\hline 7 & $\begin{array}{l}\text { Human Anatomy and } \\
\text { Physiology }\end{array}$ & Khan Academy & Gross anatomy & Self-paced & Free & No & $\begin{array}{l}\text { https://www.khanacademy.org/ } \\
\text { science/health-and- } \\
\text { medicine/human-anatomy- } \\
\text { and-physiology }\end{array}$ \\
\hline 8 & TeachMeAnatomy & TeachMeSeries team & Gross anatomy & Self-paced & $\begin{array}{l}\text { USD72/ } \\
\text { year }\end{array}$ & No & https://teachmeanatomy.info/ \\
\hline 9 & Clinical Anatomy & $\begin{array}{l}\text { Claudia Krebs, Monika } \\
\text { Fejtek and Alexa Mord- } \\
\text { horst, University of British } \\
\text { Columbia }\end{array}$ & Gross anatomy & Self-paced & Free & No & http://clinicalanatomy.ca/ \\
\hline 10 & $\begin{array}{l}\text { How to Remember } \\
\text { Anatomy for a } \\
\text { Lifetime }\end{array}$ & $\begin{array}{lrr}\text { Ayman } & \text { Taji, Internal } \\
\text { Medicine } & \text { Resident, Al- } \\
\text { Mouwasat } & \text { University } \\
\text { Hospital, Damascus, Syria }\end{array}$ & $\begin{array}{l}\text { Gross anatomy } \\
\text { and } \\
\text { neuroanatomy }\end{array}$ & $\begin{array}{l}36 \text { lectures: } \\
\text { Self-paced }\end{array}$ & USD10 & Yes & $\begin{array}{l}\text { https://www.udemy.com/course/ } \\
\text { how-to-remember-anatomy- } \\
\text { for-a-lifetime/ }\end{array}$ \\
\hline 11 & $\begin{array}{l}\text { Instant Anatomy: A } \\
\text { Course in Human } \\
\text { Anatomy }\end{array}$ & Robert Whitaker & $\begin{array}{c}\text { Gross anatomy } \\
\text { and } \\
\text { neuroanatomy }\end{array}$ & Self-paced & USD43 & Yes & $\begin{array}{l}\text { https://www.udemy.com/ } \\
\text { course/instantanatomy/ }\end{array}$ \\
\hline 12 & Medical Anatomy & $\begin{array}{l}\text { Piyank Joshi, Anatomic and } \\
\text { Clinical Pathology, Harvard } \\
\text { Medical School }\end{array}$ & Gross anatomy & $\begin{array}{l}23 \text { lectures: } \\
\text { self-paced }\end{array}$ & Free & Yes & $\begin{array}{l}\text { https://www.udemy.com/course/ } \\
\text { medical-anatomy/ }\end{array}$ \\
\hline 13 & $\begin{array}{l}\text { Anatomy of the Chest, } \\
\text { Abdomen, and Pelvis }\end{array}$ & Yale School of Medicine & $\begin{array}{l}\text { Thorax + } \\
\text { abdomen }\end{array}$ & 4 weeks & Free & Yes & $\begin{array}{l}\text { https://www.coursera.org/learn/ } \\
\text { trunk-anatomy }\end{array}$ \\
\hline 14 & $\begin{array}{l}\text { Anatomy } \\
\text { Specialization }\end{array}$ & University of Michigan & System wise & 2 months & Free & Yes & $\begin{array}{l}\text { https://www.coursera.org/ } \\
\text { specializations/anatomy }\end{array}$ \\
\hline 15 & $\begin{array}{l}\text { Anatomy: Know Your } \\
\text { Abdomen }\end{array}$ & $\begin{array}{l}\text { James Pickering, University } \\
\text { of Leeds }\end{array}$ & Abdomen & 2 weeks & Free & USD99 & $\begin{array}{l}\text { https://www.futurelearn.com/ } \\
\text { courses/anatomy-know-your- } \\
\text { abdomen }\end{array}$ \\
\hline 16 & $\begin{array}{l}\text { Anatomy of the } \\
\text { Abdomen and Pelvis; } \\
\text { a Journey from Basis } \\
\text { to Clinic. }\end{array}$ & Leiden University & Abdomen & 7 weeks & Free & Yes & $\begin{array}{l}\text { https://www.coursera.org/learn/ } \\
\text { abdomen-anatomy }\end{array}$ \\
\hline 17 & $\begin{array}{l}\text { Human Anatomy: } \\
\text { Musculoskeletal } \\
\text { Cases }\end{array}$ & Harvard University & $\begin{array}{l}\text { Musculoskeletal } \\
\text { system }\end{array}$ & 4 weeks & Free & USD144 & $\begin{array}{l}\text { https://www.edx.org/course/ } \\
\text { anatomyx-musculoskeletal- } \\
\text { cases }\end{array}$ \\
\hline 18 & Proceum - Medvizz & $\begin{array}{l}\text { Dr. G. Bhanu Prakash Kulka- } \\
\text { rni, Proceum Pvt. Ltd. }\end{array}$ & All & Self-paced & USD150 & No & $\begin{array}{l}\text { https://medvizz.com/shop/Gross } \\
\text { Anatomy }\end{array}$ \\
\hline 19 & Medical Anatomy & $\begin{array}{l}\text { Dhiren Panda, Sri Sri Univer- } \\
\text { sity, Cuttack, India }\end{array}$ & Gross anatomy & $\begin{array}{l}14 \text { lectures: } \\
\text { self-paced }\end{array}$ & USD6 & No & $\begin{array}{l}\text { https://www.udemy.com/course/ } \\
\text { anatomy-medical/ }\end{array}$ \\
\hline 20 & $\begin{array}{l}\text { Human Anatomy and } \\
\text { Physiology }\end{array}$ & $\begin{array}{l}\text { Holly Trimble, Lone Star Col- } \\
\text { lege, Texas, United States }\end{array}$ & Gross anatomy & 6 Weeks & USD115 & No & $\begin{array}{l}\text { https://www.ed2go.com/mntc/ } \\
\text { online-courses/human- } \\
\text { anatomy-and-physiology/ }\end{array}$ \\
\hline
\end{tabular}


Table 2. Tabulation of YouTube channels (unstructured material) for anatomy study meeting the inclusion criteria.

\begin{tabular}{|c|c|c|c|}
\hline No & Channel name & Offered by (university or individual) & URL \\
\hline 1 & Anatomy Hub & $\begin{array}{l}\text { Dhiren Panda, Sri Sri University, Cuttack, } \\
\text { India }\end{array}$ & https://www.youtube.com/channel/UCv2VVTConfcHe5WdJp-CkzA \\
\hline 2 & Anatomy Explained & $\begin{array}{l}\text { Ankit Khandelwal, University College of } \\
\text { Medical Sciences, Delhi, India }\end{array}$ & https://www.youtube.com/channel/UCcn-ljIpBLSiNXiTG81Acxg \\
\hline 3 & $\begin{array}{l}\text { Dr. Ashraf Ramzy } \\
\text { Lectures of Anatomy }\end{array}$ & $\begin{array}{l}\text { Ashraf Ramzy, Professor of Anatomy \& } \\
\text { Embryology in Ain Shams University, Cairo, } \\
\text { Egypt \& in Hashemite University, Zarqaa, } \\
\text { Jordan. }\end{array}$ & https://www.youtube.com/channel/UCH9QK_LA7bjsy2SVYj9yEKA \\
\hline 4 & Air Anatomy & $\begin{array}{l}\text { Doris George Yohanan, Government Medical } \\
\text { College, Trivandrum, India }\end{array}$ & https://www.youtube.com/channel/UCMz5_ng3QWX1cWNaiA1fZJA \\
\hline 5 & Dr. Santosh Bhosale & $\begin{array}{l}\text { Santosh Bhosale, S.S. Institute of Medical } \\
\text { Sciences and Research Centre, Davangere, } \\
\text { India }\end{array}$ & https://www.youtube.com/user/santyzz07 \\
\hline 6 & VBS OsteoMed & $\begin{array}{l}\text { Dr. Balasubramaniyam, St. Johns Medical } \\
\text { College, Bangalore, India }\end{array}$ & https://www.youtube.com/channel/UCHoxAI5-TXNrTDqHINr3rmA \\
\hline 7 & $\begin{array}{l}\text { Anatomy for Medical } \\
\text { Students Hussain }\end{array}$ & $\begin{array}{l}\text { Tassadaq Hussain, Central Park Medical } \\
\text { College, Lahore, Pakistan }\end{array}$ & https://www.youtube.com/channel/UCXo4BBrfJALC2-k08Kbu63g \\
\hline 8 & $\begin{array}{l}\text { Life in the Womb Dr. } \\
\text { Rose }\end{array}$ & $\begin{array}{l}\text { Rose Xaviour, Government Medical College, } \\
\text { Thrissur, India }\end{array}$ & https://www.youtube.com/channel/UCOKHgUB-tHwyH_5ubf8-gLg \\
\hline 9 & $\begin{array}{l}\text { Anatomy Classes by } \\
\text { Dr. Sushant }\end{array}$ & $\begin{array}{l}\text { Sushant Swaroop Das, Maulana Azad } \\
\text { Medical College, New Delhi, India }\end{array}$ & https://www.youtube.com/channel/UClALmZkw0CRjyh2E-clAizg \\
\hline 10 & Anatomy World & $\begin{array}{l}\text { Yusuf, Al Jouf University, Al-Jawf, Saudi } \\
\text { Arabia }\end{array}$ & https://www.youtube.com/user/AnatomyWorld \\
\hline 11 & CallosumBD & $\begin{array}{l}\text { Students of Sir Salimullah Medical College, } \\
\text { Dhaka, Bangladesh }\end{array}$ & https://www.youtube.com/channel/UC-CbtHDs-TguR1X5sMIflwQ \\
\hline 12 & $\begin{array}{l}\text { Netter's Anatomy } \\
\text { Dissections }\end{array}$ & $\begin{array}{l}\text { Faculty and staff at the University of North } \\
\text { Carolina, Chapel Hill; especially O.W. } \\
\text { Henson and Noelle A. Granger }\end{array}$ & https://www.youtube.com/channel/UCh3Q_7o4P0Pn6aR3CRZ7MvQ \\
\hline 13 & Anatomy Made Easy & $\begin{array}{l}\text { Dr. Satheesha Nayak B, Melaka Manipal } \\
\text { Medical College, Manipal, India }\end{array}$ & https://www.youtube.com/channel/UCH_XyvwjrUPaVfRRv8J9fRA \\
\hline 14 & $\begin{array}{l}\text { Clinical Anatomy } \\
\text { Explained }\end{array}$ & $\begin{array}{l}\text { Dr. Peter J. Ward, West Virginia School of } \\
\text { Osteopathic Medicine, West Virginia, United } \\
\text { States }\end{array}$ & https://www.youtube.com/channel/UCff6W76rmtEP_kZHQpOCf3g \\
\hline 15 & Sanjoy Sanyal & $\begin{array}{l}\text { Sanjoy Sanyal, Professor, Department Chair, } \\
\text { Surgeon, Neuroscientist, All Saints } \\
\text { University, St Vincent and the Grenadines }\end{array}$ & https://www.youtube.com/user/sanyalsanjoy8 \\
\hline 16 & Dr. A. K. Singh & $\begin{array}{l}\text { A. K. Singh, GMC Haldwani, Uttarkhand, } \\
\text { India }\end{array}$ & https://www.youtube.com/channel/UCXiWYs7gN8qJASVMCqYDPXA \\
\hline 17 & FreeMedEducation & Free Medical Education Team & https://www.youtube.com/channel/UCTCEJCypL-cwp2EWlT5pmGg \\
\hline 18 & $\begin{array}{l}\text { 3D4Medical from } \\
\text { Elsevier }\end{array}$ & 3D4Medical team & https://www.youtube.com/channel/UC6jEF1orhgER1Nsc174FwTg \\
\hline 19 & Sam Webster & $\begin{array}{l}\text { Sam Webster, Swansea University Medical } \\
\text { School, United Kingdom }\end{array}$ & https://www.youtube.com/user/svw0001 \\
\hline 20 & Viren Kariya & $\begin{array}{l}\text { Viren Kariya, PDU Government Medical } \\
\text { College, Gujarat, India }\end{array}$ & https://www.youtube.com/channel/UCfbo8iaSdOZwyel39GMhtlQ \\
\hline 21 & Lecturio Medical & $\begin{array}{l}\text { James Pickering, University of Leeds, Leeds, } \\
\text { United Kingdom }\end{array}$ & https://www.youtube.com/channel/UCbYmF43dpGHz8gi2ugiXr0Q \\
\hline 22 & $\begin{array}{l}\text { Human Anatomy } \\
\text { Education }\end{array}$ & $\begin{array}{l}\text { Akram Abood Jaffer, College of Medicine, } \\
\text { University of Sharjah, Sharjah, United Arab } \\
\text { Emirates }\end{array}$ & https://www.youtube.com/channel/UC_jGNnK94Pbfp-LRK5w_diA \\
\hline 23 & Animated Anatomy & Fahrice Dozic, Medical University of Graz & https://www.youtube.com/user/TheAnatomyZone \\
\hline 24 & $\begin{array}{l}\text { Dr. G. Bhanu Prakash } \\
\text { Kulkarni }\end{array}$ & $\begin{array}{l}\text { G. Bhanu Prakash Kulkarni, Proceum Pvt. } \\
\text { Ltd. }\end{array}$ & https://www.youtube.com/user/doctorbhanuprakash \\
\hline 25 & $\begin{array}{l}\text { Kenhub - Learn } \\
\text { Human Anatomy }\end{array}$ & Kenhub team & https://www.youtube.com/user/kenHubCOM \\
\hline 26 & AnatomyZone & Peter de Souza and Jack Hurley & https://www.youtube.com/user/TheAnatomyZone \\
\hline 27 & Dr. Najeeb Lectures & Dr. Zeeshan Najeeb, Miami, Florida & https://www.youtube.com/user/DoctorNajeeb \\
\hline 28 & Armando Hasudungan & Armando Hasudungan, Sydney, Australia & https://www.youtube.com/user/armandohasudungan \\
\hline 29 & Nabil Ebraheim & $\begin{array}{l}\text { Dr. Nabil Ebraheim, University of Toledo } \\
\text { Medical Center, Toledo, Ohio }\end{array}$ & https://www.youtube.com/user/nabilebraheim \\
\hline 30 & Dr Mitesh Dave & Mitesh Dave, Parul University & https://www.youtube.com/channel/UC150pgqZXetQ7exFhuT2t3Q \\
\hline 31 & The Noted Anatomist & $\begin{array}{l}\text { David Morton, University of Utah School of } \\
\text { Medicine. }\end{array}$ & https://www.youtube.com/c/TheNotedAnatomist \\
\hline 32 & Dr. Jitendra Patel & $\begin{array}{l}\text { Jitendra Patel, Smt NHL Municipal Medical } \\
\text { College,Ahmedabad, India }\end{array}$ & https://www.youtube.com/channel/UCF1KCu7tuV42tFb17MwiKIw \\
\hline
\end{tabular}


Table 3. Tabulation of non-YouTube unstructured learning material including 3D models.

\begin{tabular}{|c|c|c|c|}
\hline No & Resource name & Contributors & URL \\
\hline 1 & Anatomy Atlases & $\begin{array}{l}\text { Ronald A. Bergman, College of Medicine, University of Iowa, } \\
\text { Iowa City, Iowa, USA }\end{array}$ & https://www.anatomyatlases.org/ \\
\hline 2 & MedCharts Anatomy & Thomas R. Gest and Jaye Schlesinger, ILOC Inc., New York, USA & http://anatomy.uams.edu/medcharts.html \\
\hline 3 & HHMI BioInteractive & Howard Hughes Medical Institute, Maryland, USA & $\begin{array}{l}\text { https://www.biointeractive.org/classroom- } \\
\text { resources }\end{array}$ \\
\hline 4 & TeachMeAnatomy & Oliver Jones and Team, TeachMeSeries Ltd, England \& Wales & https://teachmeanatomy.info/ \\
\hline 5 & $\begin{array}{l}\text { Anatomy Drill and Practice, } \\
\text { Principles of Anatomy and } \\
\text { Physiology, 9th Edition }\end{array}$ & $\begin{array}{l}\text { Gerard J. Tortora, Sandra Reynolds Grabowski, Wiley Student } \\
\text { Companion Site }\end{array}$ & $\begin{array}{l}\text { http://bcs.wiley.com/he- } \\
\text { bcs/Books?action=index\&bcsId=1026 } \\
\text { \&itemId=0471366927 }\end{array}$ \\
\hline 6 & The Body Online & Dennis Daniel and Benjamin Yam, Stony Brook University, USA & http://www.thebodyonline.net \\
\hline 7 & GetBodySmart & Scott Sheffield, ConceptCreators, Inc, Camas, Washington, USA & https://www.getbodysmart.com/ \\
\hline \multicolumn{4}{|c|}{ 3D models } \\
\hline 8 & $\begin{array}{l}\text { Anatomy Learning - 3D } \\
\text { Anatomy Atlas. Explore } \\
\text { Human Body in Real Time }\end{array}$ & Anatomy Learning LLC & http://anatomylearning.com/ \\
\hline 9 & Biodigital & Biodigital Inc, New York, USA & https://www.biodigital.com/ \\
\hline 10 & $\begin{array}{l}\text { Inner Body - Human } \\
\text { Anatomy }\end{array}$ & Inner Body Research Team, California, USA & https://www.innerbody.com/htm/body.html \\
\hline 11 & ZygoteBody & American Fork, Utah, USA & https://www.zygotebody.com \\
\hline
\end{tabular}

non-commercial purposes; they are available on both mobile platform (Android) and browser-based platform (WebGL). Selected 3D models are available on the AnatomyZone website as well. The most powerful and extensive interactive 3D software platform for anatomy in health and disease is the BioDigital Human which offers exhaustive resources for teachers and personal use. This is the first platform offering the human 3D model API support for the developers. A.D.A.M. Education offers similar 3D interactive learning experience as well and needs subscription to access its contents. ZygoteBody, formerly Google Body, is a free online 3D anatomy atlas.

YouTube channels Viva Voce of Anatomy, World of Anatomy and Anatomy by Dr. Vijaya had no mention of authors with affiliations and were excluded from the list. Human Anatomy, an iPhone application by Max Soderstrom, was excluded as this app provides a very superficial system wise explanation of anatomy and physiology. Similarly, the website Anatomy Arcade by Ben Crossett, Glen Waverley Secondary College in Melbourne, Australia, was also excluded for superficial content addressing to higher school level.

\section{Discussion}

The use of internet-based technology has facilitated learning in every field and medical education is no exception [33]. Student-centred online learning materials with simple and effective instructional design are the need of the hour. In the past, there has been more emphasis on the development of reusable computer-based software platforms [34]. However, with the increase in the hand-held devices and improvement in the network speed, the dependence on online learning methods is rapidly increasing.

In this study, learning resources that were available for a learner within closed networks were excluded. These learning methods are often used as blended or mixed learning along with a regular classroom engagement. The objectives of this study were to report learning resources that are completely open and can be used by anybody interested without possible entanglement with academic programs. The structured modules considered in the list presented in the study use some form of learning management system (LMS). A LMS provides for student registration, attendance, performance, progression of learning and outcomes with possible certification.

With changing roles of teachers to facilitators of learning, orientation, individual experience, and collaborative learning in an e-learning environment are the need of the hour. Within the e-learning context, a teacher should be a motivator for students and provide proper guidance in the selection of authentic learning material among plethora of learning opportunities $[35,36]$. In the same way, medical undergraduates need to learn vast concepts and all learning may not be limited to classroom environment. Since there is more emphasis on SDL in competency-based medical education (CBME), it is the need of the hour that the students know the various avenues of learning. SDL aiming at lifelong learning of medical undergraduates obviously leverages more on the internet-based learning [37]. In this context, this paper provides the necessary compilation of most of the structured and unstructured learning materials available for learning gross anatomy by medical undergraduates.

Online learning, especially during the COVID 19 pandemicinduced lockdown, involves accessing resources (content) on the Internet. The resources can be the static text web pages, simple animations, complex 3D representations, online lectures, videos demonstrating a procedure, or explaining a concept, interactive quizzes, or any similar activities. The students' attitude towards online learning is affirmative [38, 39]. Even though learning anatomy by dissecting a cadaver is irreplaceable, online learning can provide alternative learning strategies $[35,40]$. During the pandemic, subject-matter experts or universities adapted their courses, designed and 
deployed online learning resources [41].

A structured learning course or module design aligned with university curricular objectives, with a defined completion period, combined with assessments and certification shall make online learning more convenient for students. Many universities offer such modules (at least on a small scale) to its enrolled students through closed networks [42]. Such learning material is largely unavailable for students outside that specific university. In the structured learning resources listed in the present study, the videos prepared by the University of British Columbia [27] hosted on clinical anatomy website are the most comprehensive in explaining the relevant anatomy concepts. The videos are made according to adult learning principles of online design [43]. The videos are short, effective in conveying message, outcome-oriented, learner-centred and deliver basic anatomical concepts. In the similar way, many YouTube channels (Table 2), especially Human Anatomy Education, Lecturio Medical and Dr. G. Bhanu Prakash are rich in visually engaging content with clear explanation of anatomical concepts.

In the modern era of accessibility of most learning materials online and with increasing emphasis on SDL during the pandemic-induced lockdown, both teachers and students are responsible for identifying and following authentic resources [44]. Lately, the statutory bodies regulating higher education (the University Grants Commission (UGC), India) have laid guidelines for online courses and programs and encouraged universities to move a part of the academic program online; therefore, it is imperative for the individual universities to design and deploy online courses and programs. The list presented in the study may act as baseline guide for already existing structured courses (Table 1). The future course designers and content developers can build on their success stories and rectify their shortcomings.

\section{Limitations}

We have listed only online material that can be used at any time as happens in asynchronous learning. As the synchronous learning methods are usually limited to the learners of a particular medical school or university, these resources were excluded from the present study.

\section{Conclusions}

The prevailing pandemic of COVID-19 forced a stringent lockdown in many countries. Students across the globe rely on online classes for their continued studies. At these difficult times, the list presented in the study may act as guide in selecting the simplest and best teaching and learning materials for those teaching and learning anatomy in medical undergraduate courses. However, in most cases, there is no alignment with standard learning outcomes as defined by medical education regulatory authorities.

\section{Ethical Statement}

This article does not include any human participants and/or animals.

\section{Conflict of Interest}

Authors declare no conflict of interest. Authors have no direct or indirect relation to any of the material/resources/websites or other online learning materials discussed here.

\section{Financial Disclosure}

Authors have not received any funding.

\section{References}

[1] Longhurst GJ, Stone DM, Dulohery K, Scully D, Campbell T, Smith CF. Strength, weakness, opportunity, threat (SWOT) analysis of the adaptations to anatomical education in the United Kingdom and Republic of Ireland in response to the Covid-19 pandemic. Anatomical Sciences Education. 2020;13(3):301-311. Available from: https://doi.org/10.1002/ase.1967

[2] Pather N, Blyth P, Chapman JA, Dayal MR, Flack NAMS, Fogg QA, et al. Forced disruption of anatomy education in Australia and New Zealand: an acute response to the Covid-19 pandemic. Anatomical Sciences Education. 2020;13(3):284-300. Available from: https://doi.org/10.1002/ase.1968

[3] Vaona A, Banzi R, Kwag KH, Rigon G, Cereda D, Pecoraro V, et al. E-learning for health professionals. Cochrane Database of Systematic Reviews. 2018;2018(8). Available from: https://doi.org/10.1002/14651858.CD011736.pub2

[4] Lawn S, Zhi X, Morello A. An integrative review of e-learning in the delivery of self-management support training for health professionals. BMC Medical Education. 2017;17(1):183. Available from: https://doi.org/10.1186/s12909-017-1022-0

[5] Cook DA, Levinson AJ, Garside S, Dupras DM, Erwin PJ, Montori VM. Internet-based learning in the health professions. JAMA. 2008;300(10):1181. Available from: https://doi.org/10.1001/jama.300.10.1181

[6] Cook DA, Levinson AJ, Garside S, Dupras DM, Erwin PJ, Montori VM. Instructional design variations in internet-based learning for health professions education: a systematic review and meta-analysis. Academic Medicine. 2010;85(5):909-922. Available from: https://doi.org/10.1097/ACM.0b013e3181d6c319

[7] Mo P. The use of Internet for health education. Journal of Biosafety \& Health Education. 2013;1(1):1000e102. Available from: https://doi.org/10.4172/23320893.1000e102 
[8] Gill HK, Gill N, Young SD. Online technologies for health information and education: a literature review. Journal of Consumer Health On the Internet. 2013;17(2):139-150. Available from: https://doi.org/10.1080/15398285.2013.780542

[9] Williams J. Social networking applications in health care. Proceedings of the 2010 ICSE Workshop on Software Engineering in Health Care - SEHC'10. 2010. pp. 39-49. Available from: https://doi.org/10.1145/1809085.1809091

[10] Young SD. Recommendations for using online social networking technologies to reduce inaccurate online health information. Online $\mathbf{J}$ Health Allied Sci. 2011;10(2):2. Available from: https://www.ncbi.nlm.nih.gov/pmc/articles/pmid/22016578/

[11] Larreamendy-Joerns J, Leinhardt G. Going the distance with online education. Review of Educational Research. 2006;76(4):567-605. Available from: https://doi.org/10.3102/00346543076004567

[12] Kassop M. Ten ways online education matches, or surpasses, face-to-face learning [Internet]. Backbone Communications. 2010 [cited 2021 Mar 22]. Available from: http://backbonecommunications.com/news/tenways-online-education-matches-or-surpasses-face-toface-learning/

[13] Morahan-Martin JM. How internet users find, evaluate, and use online health information: a cross-cultural review. CyberPsychology \& Behavior. 2004;7(5):497-510. Available from: https://doi.org/10.1089/cpb.2004.7.497

[14] Dilliway G, Maudsley G. Patients bringing information to primary care consultations: a crosssectional (questionnaire) study of doctors' and nurses' views of its impact. Journal of Evaluation in Clinical Practice. 2008;14(4):545-547. Available from: https://doi.org/10.1111/j.1365-2753.2007.00911.x

[15] Maloney S. Accessibility, nature and quality of health information on the Internet: a survey on osteoarthritis. Rheumatology. 2005;44(3):382-385. Available from: https://doi.org/10.1093/rheumatology/keh498

[16] Judd T, Elliott K. Selection and use of online learning resources by first-year medical students: cross-sectional study. JMIR Medical Education. 2017;3(2):e17. Available from: https://doi.org/10.2196/mededu.7382

[17] MHRD. Nptel, online courses and certification, learn for free [Internet]. A Project funded by MHRD, Govt. of India. 2021 [cited 2021 Mar 22]. Available from: https://nptel.ac.in/
[18] Alsup K, Fox GM, Sullivan KA. University of Michigan, XSeries program in anatomy. Learn the foundations of human anatomy [Internet]. Coursera. 2021 [cited 2021 Mar 9]. Available from: https://www.edx.org/xseries/anatomy

[19] Alsup K, Fox GM, Sullivan KA. Anatomy specialization [Internet]. Coursera. 2021 [cited 2021 Mar 10]. Available from: https://www.coursera.org/specializations/anatomy

[20] Yuen J, Lau B, Cheong A, Li R. Human anatomy by HKPolyUx [Internet]. edX. 2021. [cited 2021 Mar 9]. Available from: https://www.edx.org/course/humananatomy

[21] Harvard University. AnatomyX: musculoskeletal cases [Internet]. edX. 2021 [cited 2021 Mar 10]. Available from: https://www.edx.org/course/anatomyxmusculoskeletal-cases

[22] De Ruiter M. Anatomy of the abdomen and pelvis; a journey from basis to clinic [Internet]. Coursera. 2021 [cited 2021 Mar 10]. Available from: https://www.coursera.org/learn/abdomen-anatomy

[23] Norman W. The anatomy lesson [Internet]. 1999 [cited 2021 Mar 9]. Available from: http://www.wesnorman.com/

[24] TeachMeAnatomy - making anatomy simple [Internet]. 2021 [cited 2021 Mar 9]. Available from: https://teachmeanatomy.info/

[25] Dijksman JA, Khan S. Khan Academy: the world's free virtual school. American Physical Society, APS March Meeting 2011, March 21-25. 2011;A14.006. Available from: http://meetings.aps.org/link/BAPS.2011.MAR.A14.6

[26] Duncan C, Stewart WB, Kapadia S. Anatomy of the chest, abdomen, and pelvis from University of Yale [Internet]. Coursera. 2021 [cited 2021 Mar 10]. Available from: https://www.coursera.org/learn/trunk-anatomy

[27] Kenhub. Learn anatomy with Kenhub [Internet]. Kenhub. 2021 [cited 2021 Mar 10]. Available from: https://www.kenhub.com/en

[28] Pickering J. Anatomy: know your abdomen - online course [Internet]. FutureLearn. 2020 [cited 2021 Mar 10]. Available from: https://www.futurelearn.com/courses/anatomy-knowyour-abdomen

[29] Krebs C, Fejtek M, Mordhorst A. Clinical anatomy home [Internet]. Clinical Anatomy, University of British Columbia. 2020 [cited 2021 Mar 10]. Available from: http://clinicalanatomy.ca/ 
[30] Whitaker R. Instant anatomy: a course in human anatomy [Internet]. Udemy. 2015 [cited 2021 Mar 10]. Available from: https://www.udemy.com/course/instantanatomy/

[31] Taji A. How to remember anatomy for a lifetime! [Internet]. Udemy. 2017 [cited 2021 Mar 10]. Available from: https://www.udemy.com/course/how-toremember-anatomy-for-a-lifetime/

[32] Kulkarni GBP. Medvizz [Internet]. Proceum Pvt Ltd. 2021 [cited 2021 Mar 10]. Available from: https://www.medvizz.com/

[33] Dhir SK, Verma D, Batta M, Mishra D. Elearning in medical education in India. Indian Pediatrics. 2017;54(10):871-877. Available from: https://doi.org/10.1007/s13312-017-1152-9

[34] Taveira-Gomes T, Ferreira P, Taveira-Gomes I, Severo M, Ferreira MA. What are we looking for in computer-based learning interventions in medical education? A systematic review. Journal of Medical Internet Research. 2016;18(8):e204. Available from: https://doi.org/10.2196/jmir.5461

[35] Totlis T, Tishukov M, Piagkou M, Kostares M, Natsis K. Online educational methods vs. traditional teaching of anatomy during the COVID-19 pandemic. Anatomy \& Cell Biology. 2021. Available from: https://doi.org/10.5115/acb.21.006

[36] Yousuf MS, Harvey HL, Ramzy A, Al ShareiAS, Al-Zboun WQ, Badran DH. The attitude of medical students towards the teaching of anatomy. Eur J Anat. 2020;24(6):449-458. Available from: https://eurjanat.com/v1/journal/paper.php?id=200078my

[37] Premkumar K, Vinod E, Sathishkumar S, Pulimood AB, Umaefulam V, Prasanna Samuel P, et al. Self-directed learning readiness of Indian medical students: a mixed method study. BMC Medical Education. 2018;18(1):134. Available from: https://doi.org/10.1186/s12909-0181244-9

[38] Calcagnile T, Sighinolfi MC, Sarchi L, Assumma S, Filippi B, Bonfante G, et al. COVID-19 and slowdown of residents' activity: Feedback from a novel e- learning event and overview of the literature. Urologia Journal. 2021;039156032110012. Available from: https://doi.org/10.1177/03915603211001253

[39] Wolniczak E, Roskoden T, Rothkötter H-J, Storsberg SD. Course of macroscopic anatomy in Magdeburg under pandemic conditions. GMS J Med Educ. 2020;37:Doc65. Available from: https://www.egms.de/static/en/journals/zma/202037/zma001358.shtml

[40] Banovac I, Katavić V, Blažević A, Bičanić I, Hladnik A, Kovačić $\mathrm{N}$, et al. The anatomy lesson of the SARS-CoV-2 pandemic: irreplaceable tradition (cadaver work) and new didactics of digital technology. Croatian Medical Journal. 2021;62(2):173-186. Available from: https://doi.org/10.3325/cmj.2021.62.173

[41] Harmon DJ, Attardi SM, Barremkala M, Bentley DC, Brown KM, Dennis JF, et al. An analysis of anatomy education before and during Covid-19: May-August 2020. Anatomical Sciences Education. 2021;14(2):132-147. Available from: https://doi.org/10.1002/ase.2051

[42] Chang Chan AY-C, Custers EJFM, van Leeuwen MS, Bleys RLAW, ten Cate O. Does an additional online anatomy course improve performance of medical students on gross anatomy examinations? Medical Science Educator. 2019;29(3):697-707. Available from: https://doi.org/10.1007/s40670-019-00751-z

[43] Cercone K. Characteristics of adult learners with implications for online learning design. AACE Review. 2008;16(2):137-159. Available from: https://www.learntechlib.org/primary/p/24286/

[44] Jones DG. Anatomy in a post-Covid-19 World: tracing a new trajectory. Anatomical Sciences Education. 2021;14(2):148-153. Available from: https://doi.org/10.1002/ase.2054

Received: 2021-07-19

Revised: 2021-08-03

Accepted: 2021-08-10 